\title{
Multidimensional gas chromatography and its applications in food and environmental analysis
}

\author{
Alexandra Pažitná, Nikoleta Jánošková, Ivan Špánik \\ Institute of Analytical Chemistry, Faculty of Chemical and Food Technology, \\ Slovak University of Technology in Bratislava, Radlinského 9, SK-812 37 Bratislava, Slovakia \\ ivan.spanik@stuba.sk
}

\begin{abstract}
This review deals with overview of methods of multidimensional gas chromatography (MDGC), the classical meaning- conventional heart-cut MDGG, and the comprehensive two-dimensional gas chromatography $(\mathrm{GC} \times \mathrm{GC})$. MDGC is widely used because it increases required separation efficiency, which cannot be achieved by one-dimensional gas chromatography. Selected applications in food quality and safety, monitoring of environment, food authentication are summarized. This review summarizes the advances and applications of MDGC that have been published over last 10 years.
\end{abstract}

Keywords: gas chromatography, comprehensive two-dimensional gas chromatography, heart-cut multidimensional gas chromatography

\section{Introduction}

Gas chromatography (GC) is one of the most frequently used analytical methods for separation of volatile and semi-volatile organic compounds. However, one dimensional GC has a limited possibility to achieve satisfactory separation efficiency when separation of compounds in complicated matrices is required. This lack of separation efficiency can be overcome by using of two dimensional approaches, in which two independent GC ovens equipped with proper switching system and column setup are used. Separation in such systems is realized by using (a) two columns with different polarity which are connected in series (multi column chromatography) - the whole sample is eluting from the first to the second column, (b) two columns with different polarity connected in series and satisfy the conditions of orthogonality $(G C \times G C)$; in this case the whole sample elute from the first column to the second column in some intervals, $(c)$ and by using techniques, where only a small part of the sample elute to the second column $=$ backflash, foreflash and heart-cut . Backflash is a method, where after elution of the portions of sample into the second column, the rest is washed from the first column by switching the direction of carrier gas flow to opposite direction. Foreflash is used for removing the excess of solvent, derivatization agent or other additives. Heart-cut allows transfer of one or more fractions from the first dimension to the second dimension with another polarity. Transfer to the second dimension is carried out by an on-line cutting, which allows transfer for only a certain analytes (de Alencastro et al. 2003). The technique is employed to improve the resolution of a complex mixture. An example of this procedure might be the measurement of the contaminant benzene in a complex hydrocarbon mixture such as gasoline. In a multi-component hydrocarbon mixture a trace of benzene will be masked by other co-eluting substances with all types of stationary phase. It follows that the separation must be carried out employing two types of stationary phase, one dispersive and the other polar. After this fulfillment of conditions two peaks with better resolution will be obtained (Fig. 1).

The most basic and also the cheapest way how to realize two-dimensional experiment is to locate both columns with different stationary phases into one GC oven by using two independent detectors and a switching system. Disadvantage of this system is relatively small system flexibility due to the possibility of using only one temperature program for both separation dimensions. The second option is to work with two independent GC ovens, where compounds are firstly separated in the first column. At the time of elution of retained compounds suitable switching system switches the flow of mobile gas to
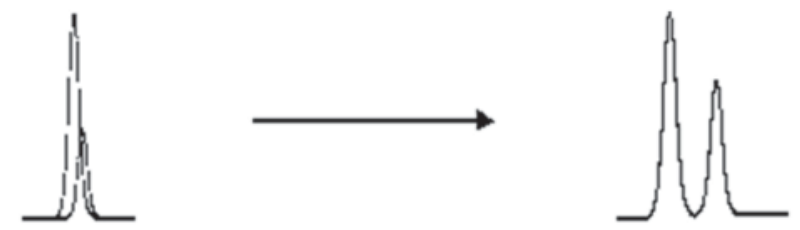

Fig. 1. Heart cutting for improved resolution. Overloading of peaks after separation in the first dimension (left) and resolved peaks after separation in the second dimension (right) on column with another polarity. 
the second column, where these compounds are divided into individual components and possibly their concentration is determined (de Alencastro et al. 2003).

The resolution in MDGC system is determined by the column(s) dimensions and the difference in separation power between the two stationary phases. The separation can be improved by using of longer columns and smaller internal diameters. The difference in the selectivity between the two stationary phases will strongly influence the final separation; e.g., increasing the difference in polarity may be expected to improve the separation.

All types of detectors (e.g. flame ionization detector (FID), electron capture detector (ECD), atomicemission detector (AED), nitrogen-phosphorus detector (NPD), olfactory detector and mass spectrometer (MS)) can be used for two-dimensional GC. Increased demands are placed on the sample treatment methods where the presence of impurities often causes changes in retention times and is quite difficult to properly adjust cutting of the desired compound into the second column. Standards are used for confirmation the accuracy of cutting time.

\section{Applications of convential heart-cut mdgc system}

The first demonstration of MDGC was published by Simmons and Snyder (Simmons and Snyder 1958) in 1958. Tranchida reviewed the history of heart-cutting two-dimensional GC (Tranchida et al. 2012). MDGC system with a switching device is shown in the Fig. 2. The sample is injected into the primary column via a GC inlet. There is a switching device between two columns, which allows transport of the analyte from the first to the second dimension. Immediately, when the compound of the interest is eluting from the first column, the flow of carrier gas is switched into the second column, where separation takes place. After this procedure, the switching device is switched back to the original position (Lewis 2002). A basic principle of pressures switching was firstly described by Deans (Deans 1968). In this process are highlighted three distinct phases, that of survey of prefractionation, sample transfer and backflash of the primary column (Bertsch 1990). Method is based on the retraction of flows by using pressure balancing at intersections. The flow of carrier gas to each intersection is controlled by solenoid valves.

MDGC is used for analytical separation of complicated matrices, when the detection of minor compounds or hardly processable compounds is required. This method offer a good separation efficiency, selectivity and retention factors which are needed to achieve chromatographic resolution of target analytes present at extremely low concentration levels.

\section{Food analysis}

One of the examples of using MDGC is separation of enantiomers of volatile organic compounds, e.g. those contained in mandarin essential oil (Sciarrone et al. 2010). This analysis was performed by using one dimensional GC with chiral column connected to flame ionization detector and results were compared by heart-cut MDGC results. The separation system consists of two GG ovens, which were connected together, FID detector in the first and MS in the second dimension. A 118 Italian mandarin oils were tested in SLB-5ms stationary phase in the first dimension and for separation of enantiomers of $\alpha$-thujene, $\alpha$-pinene, camphene, $\beta$-pinene, sabinene, $\alpha$-phellandrene, $\beta$-phellandrene, limonene, linalool, citronellal, terpinen-4-ol and $\alpha$-terpineol a Megadex DETTBS- $\beta$ (2,3-diethyl-6-tert-butylsilyl -cyclodextrine) stationary phase in second dimension was used. It can be said, that the same enantiomer ratio was observed for $(+)$ - $\alpha$-thujene, (+)-sabinene, (-)- $\alpha$-phellandrene, (+)-limonene, (-)-terpinen-4-ol for both used methods. The biggest difference in enantiomer ratios identified only by one dimensional GC was reported for $(+)$-camphene and (+)-linalool. (-)- $\alpha$-pinene, while the enantiomer ratio of (-)-camphor and (+)-citronellal has been detected only by MDGC. Natural products often contain chiral compounds which enantiomeric purity depends on their origin. Determination of

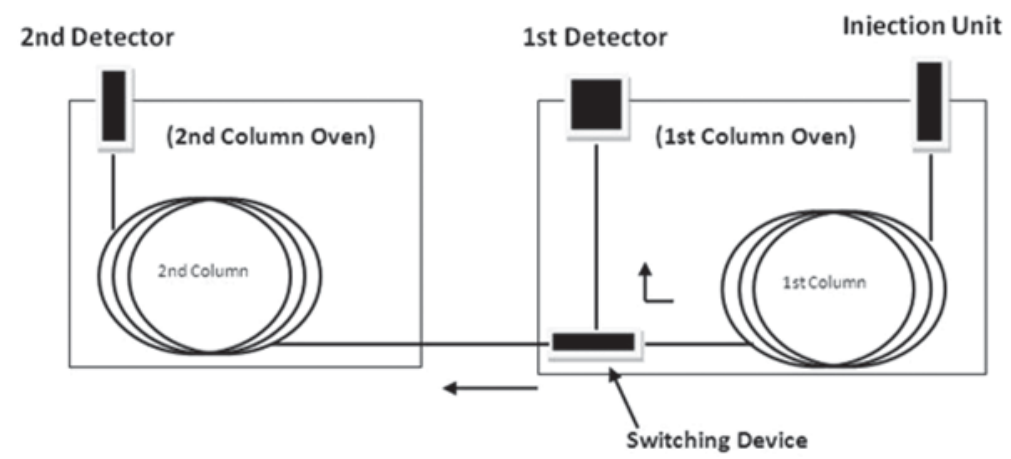

Fig. 2. MDGC system with a switching device, two GC ovens and two detectors. 
enantiomer ratios is a way how to characterize the origin of the samples and the possible adulteration. Given the complexity of natural oils, chiral compounds often need to be isolated from the others in the first non-chiral column before distribution in the chiral column of MDGC system. In F. Begnaud and A. Chaintreau work (Begnaud and Chaintreau 2005), MDGC was used to confirm authenticity of natural bergamot oil using enentiomer ratio of linalool. Expected enantiomer ratio in natural bergamot oil is $0.5 / 99.5-\mathrm{S} / \mathrm{R}$, while any changes in this ratio may be caused by illicit adulteration. Among other MDGC applications can also be included the separation of enantiomers of volatile organic compounds in juniper flavored spirits, where enantiomers of organic compounds from samples from Slovakia, Belgium, Great Britain, Germany and Czech Republic were separated. A solid phase microextraction (SPME) and also liquid-liquid extraction (LLE) were used as a sample treatment method. MDGC system consists of two independent GC in which the MS detector is in the first and FID in the second dimension. In this system, volatile organic compounds were firstly separated at nonchiral DB-FFAP column. Chiral volatile organic compounds (VOC) present at the highest concentration levels (e.g. $\alpha$-pinene, limonene, linalool oxides, linalool and 4-terpineol) were separated into their enantiomers in the second dimension using chiral (Chirasil- $\beta$-Dex) stationary phase. The significant differences in enantiomer ratios were observed for cis-linalool oxide in samples that originate from Germany and Great Britain. Samples that originate from Slovakia can be distinguished from samples from Belgium based on differences in enantiomer ratio for limonene. The conclusion of this work was finding that enantiomer ratios depend on country of their origin which could be in tight connection with technology used for their production (Pažitná et al. 2011). The distribution of enantiomers of selected chiral volatile organic compounds in 45 monofloral honey samples was studied by GC. The volatile organic compounds were extracted from Slovakian rapeseed, acacia, sunflower basswood and raspberry honeys by SPME. The isomeric ratios of linalool, cis- and trans-furanoid linalool oxides, hotrienol and four isomers of lilac aldehydes were determined. It was found that distribution of enantiomers in honey samples partially depended on their botanical origin. The differences in ratios of lilac aldehyde isomer $\mathrm{B}$ and hotrienol were observed for acacia honey that allowed us to distinguish this type of honey from others. Similarly, a different isomeric ratio of trans-furanoid linalool oxide was found for sunflower honeys (Pažitná et al. 2012). Other work studied separation of chiral monoterpenoids in pep- permint oil. Enantiomer ratio of these compounds is an important indicator of authenticity of this oil. 16 samples of peppermint oil were analyzed and enantiomer ratios were determined for menthon, neomenthol, isomenthon, menthol, neoisomenthol and menthylacetate. A MDGG system with engagement of columns - non-chiral pre-column and chiral main column was used while a separation of all six chiral monoterpenoids was achieved in one chromatographic analysis. All of chiral monoterpenoids are in a true oils as a pure enantiomers in $(1 \mathrm{R})-$ configuration. This fact implies that the presence of (1S) - enantiomers of $(+)$-menthylacetate and $(+)$-menthol in commercial peppermint oils should be considered as a manifestation of falsification (Faber et al. 1994). Liu et al. focused on distribution of enantiomers of anatabine, nornicotine and anabasine in commercial tobacco (Liu et al. 2008). They compared three types of different processed tobacco and found that the enantiomer ratios of anatabine and nornicotine varied due to the use of different processing method. S-(-)-anatabine, as a proportion of total anatabine, was $86.6 \%$ for flue-cured, $86.0 \%$ for burley and $77.5 \%$ for oriental tobacco. S-(-)-nornicotine, as a proportion of total nornicotine, was $90.8 \%$ in oriental tobacco and higher than in burley $(69.4 \%)$ and flue-cured $(58.7 \%)$ tobacco. S-(-)-anabasine, as a proportion of total anabasine, was relatively independent on used proceeding technology. Based on these facts it was possible to distinguish samples according to the type of processed tobacco. One of the many applications of MDGC in connection with SPME is a detection of falsification of olive oil (Flores et al. 2006). It is possible to determine adulteration of olive oil by hazelnut oil based on enantiomer ratio of each chiral compound and based on absence of filbertone. This method was used to distinguish olive oils adulterated with hazelnut oil.

Cullere et al. studied 24 different wines to analyze the levels of aliphatic aldehydes by using solid phase extraction and heart-cutting GC. DB-Wax column was used in the first dimension and in the second one was used VF-5. In this work Cullere and his coworkers were able to fully isolate $\mathrm{C}_{8}-\mathrm{C}_{11}$ aliphatic aldehydes in wines (Cullere et al. 2011).

Trace odor active components in wine were detected by Ochiai and Sasamoto. They used stir bar sorptive extraction as a sample treatment method. In this study, they used a preparative fraction collector to concentrate fractions of 20 injections into a single sorbent trap. After collection, the trap was thermally desorbed into the primary column of the same system for full two-dimensional analysis (Ochiai and Sasamoto 2011). A methodology for the identification of character-impact odorants in cori- 
ander leaf (Coriandrum sativum) and hop (Humulus lupulus) essential oils samples using heart-cut multidimensional gas chromatography-olfactometry (MDGC-O) was published by Eyres et al. This method is able to resolve a number of selected co-eluting odour regions and maintain one discrete peak per compound with a broader peak width, making it very suitable for olfactory assessment. In coriander leaf, only $E$-2-dodecenal was found to contribute to a co-eluting odour region, E-2-dodecen-1-ol and 1-dodecanol being present below detection threshold. Using MDGG on a hop essential oil sample, eight significant peaks were resolved from an $18 \mathrm{~s}$ heart-cut where a potent odorant was perceived during GC-O (Eyres et al. 2007). Boonlander et al. determined the enantiomeric distribution of a monoterpene alcohol linalool 3,7-dimethylocta1,6-dien-3-ol in green and roasted coffee using a polyethyleneglycol primary column followed by achiral secondary column after extraction by simultaneous distillation-extraction (SDE) and stir-bar sorptive extraction (SBSE) (Bonnlander et al 2006). Two dimensional GC technique for determining the concentration of 2-aminoacetophenone in wine was developed by Schmarr and co-workers. SPE was used as a sample preparation method. ZB-Wax column connected to FID detector was used in the first dimension and in the second one ZB-5 with MS detector. In aromatic wines such as a Muskateller, an AAP concentration of $0.8 \mu \mathrm{g} / 1$ is not recognized as UTA, whereas similar concentrations of 0.7 or $1.0 \mu \mathrm{g} / 1$ already cause UTA perception in a MüllerThurgau or Silvaner wine, respectively (Schmarr et al. 2007).

\section{Environmental analysis}

It was shown, that MDGC can be a suitable method for analyzing the organic compounds and pollutants found in water, air, soil and sediment. Heartcut method coupled to MS-MS was used to detect ultratrace levels of tributyltin in surface water samples. Tributyltin compounds are considered toxic chemicals which have negative effects on human and environment. Stir bar sorptive extraction was chosen as a sampling method. Tributyltin was detected with a triple quadrupole MS (Devos et al. 2012). Two-dimensional gas chromatography coupled to triple quadrupole mass spectrometry was also used for quantification of enantiomeric fractions (EFs) of atropisomeric polychlorinated biphenyls (PCBs). The method was successfully applied to a variety of sample types, such as soil, air, herring and human milk (Bucheli and Brandli 2006).

A chromatographic procedure for the determination of the enantiomeric fractions of o,p-DDT based on heart-cutting multidimensional gas chromatography was developed by Muñoz-Arnanz et al. The enantiomeric ratios of o,p-DDT in soil samples polluted by organohalogen compounds can be determined by using combination of an achiral (DB5) column and a chiral (BGB-172) column. The MDGC system consisted of two independent GC chromatographs, both equipped with a ${ }^{63} \mathrm{Ni}-\mathrm{ECD}$ system (Muñoz-Arnanz et al. 2009). Perez-Fernandez et al. used MDGG to separate chiral PCBs and sulfone metabolites. The sample was first separated on a nonpolar column, after than selected PCBs and metabolites were transferred into the second column which was chiral. Eanatiomeric fractions were determined for the chiral PCBs in fish oils and in cow liver (Perez-Fernandez et al. 2012). De Alencastro and co-workers presented determination of trace levels of pesticides, polychlorinated biphenyls (PCBs) and polybrominated diphenyl ethers (PBDEs) with MDGC-ECD/ECD and MDGC-ECD/MS in fish, mussels and sewage sludge. As the results showed, MDGC was surely a good technique to help the analytical chemist to solve easily problems when, analyzing complexes mixtures of environmental pollutants (de Alencastro et al. 2003).

\section{Applications of comprehensive two-dimensional gas chromatography}

Comprehensive two-dimensional gas chromatography $(\mathrm{GC} \times \mathrm{GC})($ Tranchida et al. 2009) is a special type of multidimensional gas chromatography. GC $\times$ GC was firstly described in the 90 's years of last century and it was considered as one of the most revolutionary methods of gas chromatography. The entire sample passes successively through two columns with different stationary phases. The important issue in $\mathrm{GC} \times \mathrm{GC}$ is a modulator, which connects the columns and provides a modulation of the first column effluent. As a result, 2D chromatograms with peaks showed as spots are obtained.

An orthogonality approach is generally used for selection of combination of stationary phases for $1^{\text {st }}$ and $2^{\text {nd }}$ dimension. All types of stationary phase could be successfully used in the first and second dimensions of a GC×GC system, depending on the desired analyte-stationary phase interactions. The combination of non-polar and polar columns is most popular in $\mathrm{GC} \times \mathrm{GC}$ technique. It is mostly due to available information about behavior of a huge number of compounds on non-polar columns in conventional GC, which could be easily utilized to optimize the separation in the first dimension. In this case, a separation is based on disperse interaction of analyzed compounds with stationary phase. The opposite combination (e.g. medium-polar, polar of shape-selective) is not fully 
orthogonal, since the separation is firstly governed by specific interaction(s). The main advantage of orthogonality is that order structures are disclosed in a $\mathrm{GC} \times \mathrm{GC}$ chromatogram for structurally related homologues, congeners and isomers. This fact is very convenient for performing group-type identification, since the related compounds are showing up as clusters or bands. Non-orthogonal setups are also applied in a limited number of studies, and useful for more polar analytes. A more detailed discussion of orthogonality is given in (Ryan et al. 2005). The first dimension requires to use efficient capillary columns similar to one dimensional chromatography $(15-60 \mathrm{~m} \times 0.25-0.53 \mathrm{~mm} \mathrm{ID} \times$ $\left.0.25-1 \mu \mathrm{m} \mathrm{d}_{\mathrm{f}}\right)$. Since the separation in the second dimension should be rapid and effective, short and narrow bore columns $(0.5-1.5 \mathrm{~m} \times 0.1-0.25 \mathrm{~mm}$ ID $\times 0.1-0.25 \mu \mathrm{m} \mathrm{d}_{\mathrm{f}}$ ) are applied (Wilson and Wilson's 2009). Moreover, the retention time of the compound in the second dimension should be smaller than the modulation period. In other case, the separation effectiveness of the chromatographic system is decreased, and because of this fact short second dimension column with a small internal diameter is utilized. At the same time, the problem of column overloading could occur in the case of the narrow columns. Harynuk et al. studied the influence of the second dimension internal diameter with similar phase ratios on the overloading aspect, and it was found, that columns with $250 \mu \mathrm{m}$ ID represents better choice than the smaller diameter columns for analysis of mixture of alkanes (Harynuk et al. 2005). GC $\times$ GC can be used as an effective method for determination of composition of samples in various field of scope e.g. determination of origin of various food samples, in environment, medicine (Tranchida et al. 2009) and also profiling of fats, oils and essential oils (Tranchida et al. 2004).

\section{Environmental analysis}

One of many examples of using GC $\times$ GC is study of the oil-sands (Hao et al. 2005). Oil-sand naphthenic acids (NAs) are organic waste products formed during the oil-sand digestion and extraction processes. They are very difficult to separate and determine as individual components due to their complex compositions. NAs are toxic and are a complex mixture with molecular weights $<500$, and primarily a 5 or 6 -carbon ring structure. Further investigations were devoted to optimization of the GC separation parameters for the other homologs of naphthenic acids. Moreover, gel permeation chromatography fractionation is a perspective approach to simplifying the $\mathrm{GC} \times \mathrm{GC}$ separation and identification of toxic components. Leo L.P. van
Stee applied GC $\times$ GC in combination with atomic emission detector for determination of aliphatic and aromatic thiols, alkylated benzothiophenols (BTS), dibenzothiophenols (BNTs) in crude oil and oil modified by fluidized catalyst cracking. Oil contains alkanes and alkylated cyclic compounds depending on its source. Dibenzothiophenes and benzonaphthothiophenes were identified in crude oil, while they were absent in the modified oil where alkyl aromatics (C1-C6) prevails (Stee et al. 2003). Another application of $\mathrm{GC} \times \mathrm{GC}$ is determination of volatile fractions extracted from the wooden rail supports modified by impregnation with creosote (Mateus et al. 2008). Creosote, a distillation product of coal tar, is oily liquid, amber to black in color, and is one of the most widely used wood preservatives in the world. Rail supports were recycled and wood with creosote was burned which resulted into release of toxic substances to the environment. With respect to its chemical composition, creosote is a variable and highly complex mixture that can contain up to $85 \%$ of polycyclic aromatic hydrocarbons (PAHs) together with $10 \%$ phenolic and $5 \%$ N-, S-, O-heterocyclic compounds. The mixture of creosote includes around 10,000 chemicals, but only 300 have been identified so far. The majority of the identified chemicals in the mixture can be classified as toxic and mutagenic, and related to harmful health effects. Volatile fractions were obtained by extraction with a mixture of pentane/diethyl ether 1:1. The extract was analyzed by using GC-qMS, GC $\times$ GC-qMS, GC $\times$ GC-TOFMS and GC $\times$ GC-NPD. Phenols, derivatives of benzene, PAHs (derivatives of indene, fluorene, anthracene, phenanthrene, naphthalene, and biphenyl), heterocyclic compounds, saturated and unsaturated, branched and linear hydrocarbons, alcohols and esters were identified in the extract. Samples of creosote contain nitrogen heterocycles, which are highly toxic substances, present even in low concentrations. Zapadlo et al. devoted their study to the identification of polychlorinated biphenyls (PCBs) using comprehensive two-dimensional gas chromatography coupled to time-of-flight mass spectrometer with ionic liquids SLB-IL-59 (1,12-di-(tripropylphosphonium) dodecane bis(trifluoromethan-sulfonyl) as stationary phases in the second dimension. A complex mixture of PCBs with possible congeners 150209 could be found in environmental samples. PCB standards and two real samples of Aroclor 1242 and the Aroclor 1260 were analyzed. GC $\times$ GC separated a 196 individual $\mathrm{PCBs}$ congeners, 5 peaks contain 2 PCBs and 1 peak containing 3 PCB congeners. All relevant EPA toxic PCBs were separated while two PCB congeners (CB12, CB13) were separated only in standard mixtures and the separation was 
not achieved in real samples. This indicates a strong matrix effect which must be taken into account when real samples are analyzed (Zapadlo et al. 2010). Moraes et al. analyzed the chemical composition of the volatile compounds produced during the pyrolysis of sugar cane straw (SCS). The original biomass and the residual solid phase were previously investigated by thermogravimetric Analysis and Fourier Transform Infrared Spectroscopy. The thermogravimetry showed profile similar to cellulose and, according to FTIR analysis, a presence of number of oxygenated compounds was confirmed, whereas the solid residue was mainly composed by silica. In addition, GC $\times$ GC-TOFMS technique provided the identification of 123 compounds, mainly oxygenated (acids, ethers, aldehydes, ketones and phenols) with a predominance of furfural and hexenoic acid in bio-oil (Moraes et al. 2012).

\section{Food analysis}

Separation of monoterpenoids from grape by HS-SPME-GC $\times$ GC-TOFMS was used to determine their origin (Rocha et al. 2007). More than 56 monoterpenoids were identified, while 20 of them were identified in grapes first time. Except of monoterpenes, also hydrocarbons, oxides, alcohols, aldehydes, esters and acids were found. This method was later applied for the identification of different grape varieties, since it was supposed that they have different distribution of monoterpenes.

Enhanced analytical characteristics were obtained for analysis of 16 organophosphorus pesticides (OPs) in food matrices by comprehensive twodimensional gas chromatography coupled with a flame photometric detector $(\mathrm{GC} \times \mathrm{GC}-\mathrm{FPD})$. Three pairs of co-eluting OPs in 1D GC were separated by $\mathrm{GC} \times \mathrm{GC}$ with a non-polar/polar column set (DB-5 $\times$ DB-1701), a 4s modulation period, and modulator temperature of $50{ }^{\circ} \mathrm{C}$. Six OPs were found at low concentrations $\left(0.79-5.12 \mu \mathrm{g} . \mathrm{kg}^{-1}\right)$ in 5 samples analyzed in six different food matrices. The developed method was characterized by linear calibration dependencies over the concentration range from 5 to $1000 \mu \mathrm{g} \cdot \mathrm{L}^{-1}$ with the limits of detection were $1.5-5.6 \mu \mathrm{g} . \mathrm{L}^{-1}$ for $16 \mathrm{OPs}$ ( $\mathrm{Liu}$ et al. 2012). Weldegergis et al. studied volatile substances in red wines coming out of South Africa by SPE-GC×GC-TOFMS (Weldegergis et al. 2011). Various factors affected the quality of the wine and therefore it is necessary to analyze the composition of the VOC. More than 250 VOG were identified in the wine samples. It was concluded that significant VOC (esters and alcohols) essential for wine flavor are formed mainly during fermentation. Numerically, the largest group of flavor compounds in wine was esters. Nearly 80 esters and a total of
34 alcohols were identified. Some alcohols were specific depending on grape cultivars. For example Pinotage was characteristic by presence of hexan2-ol, nonan-2-ol, dodecan-1-ol, while Cabernet Sauvignon samples were characteristic by presence of (Z)-hex-3-en-1-ol, octan-3-ol, nonane-1,9-diol. Since studied VOC are mostly presented in the samples at very low levels (in $\mu \mathrm{g} . \mathrm{L}^{-1}$ and lower), thus effective preconcentration procedure is required. Comprehensive two-dimensional gas chromatography compare to one-dimensional gas chromatography, is characterized by significant benefits, such as higher resolution and more sensitive analysis of VOC. Yang et al. applied GC $\times$ GC-TOFMS for screening of non-target pollution by halogenated compounds in the fish sample. The sample pretreatment procedure included extraction with hexane/ actone $(1: 1, V / V)$, in combination with cleaning by gel permeation chromatography with siilica gel packed column. In general, 72 compounds were identified as organohalogens, which including 33 polychlorinated biphenyls, 9 organochlorine pesticides, 4 polybrominated diphenyl ethers, 4 metabolites of DDT, 2 chlorinated anisole, 2 chlorostyrene, 1 thioanisole, and methyl triclosan. Ten dominating PCBs and one PBDE were quantified by the external standard method in 5 fish samples with 10 PCBs and BDE-47 concentration ranged from 52-332 ng. $\mathrm{g}^{-1}$ lipid and 4.8-17 ng. $\mathrm{g}^{-1}$ lipid, respectively (Yang et al. 2012).

$\mathrm{GC} \times \mathrm{GG}$ coupled with a nitrogen chemiluminescence detection system was also applied for the analysis dangerous levels of mutagenic and carcinogenic nitrosamines in meat and vegetable samples. Since the grilling of lamb on unready charcoal resulted in the formation of considerable quantities of nitrosoamines, the charcoal-grilled lamb samples at various stages of cooking and with various fat contents and also charcoal-grilled vegetables were studied. The GC $\times$ GG-NCD method showed high selectivity, sensitivity to six pure nitrisiamines standards, moreover all of the six tested nitrosoamines responded with equal nitrogen response (to within $\pm 7.3 \%$ ). Consequently, it was shown the response of the detector is independent on the molecular structure or other functionality $(\mathrm{n}=5)$, and this makes quantification procedure easier than with MS detector (Kocak et al. 2012). $\mathrm{GC} \times \mathrm{GC}-\mathrm{MS}$ was used for analysis of VOC profile of two strawberries cultivars (Selva and Adina) and for determination of differences between fresh picked and post-harvest strawberries of the same cultivar (Williams et al. 2004). VOC of fresh strawberries were extracted by SPME and the enantiomer separations were performed on chiral stationary phases. Chiral GC $\times$ GC facilitated the detection of key 
enantiomers in strawberry flavor. The (-)-enantiomer of 2,5-dimethyl-4-hydroxy-(2H)-furan-3-one (DMHF) and the S-enantiomer of linalool were tentatively identified as the predominant forms in the both cultivars Selva and Adina. The effects of post-harvest storage were disclosed, since the postharvested fruits were characterized with increased levels of DMHF and nerolidol, and reduced levels of benzaldehyde and methyl hexanoate compared to fresh samples. A number of unknown analytes in the volatile profiles were detected by using $\mathrm{GC} \times \mathrm{GC}$, and thus (chiral) GC $\times$ GC-MS analysis is recommended for future investigations for the improved volatile characterization of strawberry cultivars. $\mathrm{GC} \times \mathrm{GC}$ was also applied for the determination of flavor compounds in butter (Adahchour et al. 2005). One dimensional GC has showed significant effect of heat treatment, when concentrations of maltol and furaneol has increased, while the concentrations of the vanillin remains at the same level. In addition, a high separation efficiency of $\mathrm{GC} \times \mathrm{GC}$ was used to determine the unknown groups of substances in real samples - aldehydes, ketones, alcohols, fatty acids, and lactones. Some of furan and heterocyclic compounds derivatives (emerging at temperatures over $150{ }^{\circ} \mathrm{C}$ ) were identified only in the samples after estensive heat treatment, while others, like furaneol or diacetyl were found in the samples before and also after heat treatment.

In order to provide more accurate identification of multiclass pesticides along with improved sensitivity, Khummueng et al. utilized the $\mathrm{GC} \times \mathrm{GC}$ with dual-detection system (NPD and ECD) for analysis of spinach extract (Khummueng et al. 2008). Rochat et al. applied GC $\times$ GC-TOFMS for analysis of S-containing compounds in roasted beef as key aroma components. Six identified Scompounds were parallel detected with GC-SNIF (olfactometry), whereas the presence of some compounds was only confirmed by TOFMS (Rocha et al. 2006).

\section{Other analysis}

The enhanced separation of GC×GC overcomes the problem of poor separation in the case of drug analysis, which leads to wrong or misinterpreted results (Kueh et al. 2003).

Silva Jr. et al. demonstrated the capability of GC $\times$ GC-TOFMS for screening of five key illicit compounds at low concentration levels in urine samples. Furthermore, the received results fulfilled the identification criteria set by World Anti-Doping Agency (WADA) (Silva Jr. et al. 2009). The main benefit of application of $\mathrm{GC} \times \mathrm{GC}$ coupled with TOFMS for doping control is connected with improved peaks deconvolution. Mitrevski et al. compared two column sets $(\mathrm{NP} / \mathrm{P}$ and $\mathrm{P} / \mathrm{NP}$, $\mathrm{NP}=\mathrm{BPX} 5 ; \mathrm{P}=\mathrm{BPX} 50)$ to maximize sensitivity and achieve WADA-analysis criteria for determination of sterols in urine samples. It was shown that the $\mathrm{NP} / \mathrm{P}$ combination offered better separation, while the $\mathrm{P} / \mathrm{NP}$ set provided better peak shape and sensitivity (Mitrevski et al. 2011). The identification of compounds were supported by an in-house library and predefined $1 \mathrm{D}$ and 2D retention times for peak confirmation (Mitrevski et. al. 2008).

In the further investigation, Guthery et al. used GC $\times$ GC to analysis of human serum spiked with the benzodiazepines (diazepam, nordiazepam, bromazepam, oxazepam, temazepam, lorazepam, flunitrazepam and 7-aminoflunitrazepam) and street heroin (heroin, 6-onoacetylmorphine, morphine, acetylcodeine and papaverine). The combination of opiates and benzodiazepines were chosen as these compounds have very similar chemical and physical properties, and difficult separation. Moreover, some endogenous compounds were identified, and nearly 1700 peaks were detected in the samples. The sensitivity and repeatability was estimated at concentration range 5-1000 ng. $\mathrm{ml}^{-1}$. The limits of detection and quantification were 1.6 and $5.4 \mathrm{ng} \cdot \mathrm{ml}^{-1}$ for flunitrazepam, and 2.5 and $8.5 \mathrm{ng} . \mathrm{ml}^{-1}$ for 7 -aminoflunitrazepam. All studied compounds were effectively separated and identified by GC×GC-TOFMS (Guthery et al. 2009).

\section{Acknowledgement}

This work was supported by VEGA grant no. 1/0972/12. Alexandra Pažitná would like to thank Slovak University of Technology for grant supporting young researchers.

\section{References}

Adahchour M, Wiewel J, Verdel R, Vreuls RJJ, Brinkman UST (2005) Journal of Chromatography A 1086: 99-106.

Alencastro LF, Grandjea D, Tarradellas J (2003) Chimia 57: 499-504.

Begnaud F, Chaintreau A (2005) Journal of Chromatography A 1071: 13-20.

Bertsch W (1990) Chromatogtraphic Science Series 50: 74-144.

Bonnlander B, Cappuccio R, Liverani FS, Winterhalter P (2006) Flavour and Fragrance Journal 21: 637-641.

Bucheli TD, Brandli RC (2006) Journal of Chromatography A 1110: 156-164.

Cullere L, Ferreira V, Cacho J (2011) Food Chemistry 127: 1397-1403.

Deans RR (1968) Chromatographia 18: 18-22.

Devos C, David F, Sandra P (2012) Journal of Chromatography A 1261: 151-157.

Eyres G, Marriot PJ, Dufuor JP (2007) Journal of Chromatography A 1150: 70-77.

Faber B, Dietrich A, Mosandl A (1994) Journal of Chromatography A 666: 161-165. 
Flores G, del Castillo R, Blanch ML, Herraiz GP (2006) Food Chemistry 97: 336-342.

Guthery B, Bassindale Al, Pillinger CT, Morgan GH (2009) Rapid communnications in mass spectrometry 23: 340-348.

Hao Ch, Headley JV, Peru KM, Frank R, Yang P, Solomon KR (2005) Journal of Chromatography A 1067: 277-284.

Harynuk J, Górecki T, de Zeeuw J (2005) Journal of Chromatography A 1071: 21-27.

Khummueng W, Morrison P, Marriott PJ (2008) Journal of Separation Science 31: 3404-3415.

Kocak D, Oze MZ, Gogus F, Hamilton JF, Lewis AC (2012) Food Chemistry 135: 2215-2220.

Kueh AJ, Marriott PJ, Wynne PM, Vine JH (2003) Journal of Chromatography A 1000: 109-124.

Lewis AC (2002) Multidimensional High Resolution Gas Chromatography, In: Multidimensional Chromatography, Mondello, L.; Lewis, A.C.; Bartle K.D. (Eds), pp. 47-75, John Wiley \& Sons, Ltd, ISBN 0-47198869-3, Chichester, England.

Liu B, Chen Ch, Su DWQ (2008) Journal of Chromatography B 865: 13-17.

Liu X, Mitrevski B, Li D, Li J, Marriott PJ (2012) Microchemical Journal (in the press)

Mateus EP, Gomes da Silva MDR, Ribeiro AB, Marriott PJ (2008) Journal of Chromatography A 1178: 215-222.

Mitrevski B, Veleska B, Engel E, Wynne P, Song SM, Marriott PJ (2011) Forensic Science International 209: $11-20$.

Mitrevski BS, Brenna JT, Zhang Y, Marriott PJ (2008) Journal of Chromatography A 1214: 134-142.

Moraes MSA, Georges F, Almeida SR, Damasceno FC, Maciel GPS, Zini CA, Jacques RA, Caramão EB (2012) Fuel Processing Technology 101: 35-43.

Muñoz-Arnanz J, Bosch C, Fernandez P, Grimalt JO, Jimenez B (2009) Journal of Chromatography A 1216 : 6141-6145.

Ochiai N, Sasamoto KJ (2011) Journal of Chromatography A 1218: 3180-3185.

Pažitná A, Janáčová T, Špánik I (2011) 35th International Symposium on Capillary Chromatography and 8th GC $\times$ GC Symposium, San Diego, USA, Book of Abstracts L-03-38.
Pažitná A, Janáčová T, Špánik I (2012) Journal of Food and Nutrition Research 51: 236-241.

Perez-Fernandez V, Castro-Puyana M, Gonzalez MJ, Marina ML, Garcia MA, Gomara B (2012) Chirality 24: 577-583.

Rocha SM, Coelho E, Zrostlíková J, Delgadillo I, Coimbra MA (2007) Journal of Chromatography A 1161: 292-299.

Rochat S, Saint Laumer J-Y, Chaintreau A (2007) Journal of Chromatography A 1147: 85-94.

Ryan D, Morrison P, Marriott P (2005) Journal of Chromatography A 1071: 47-53.

Sciarrone D, Schipilliti L, Ragonese C, Tranchida PQ, Dugo P, Dugo G, Mondello L (2010) Journal of Chromatography A 1217: 1101-1105.

Schmarr HG, Ganß S, Sang W, Potouridis T (2007) Journal of Chromatography A 1150: 78-84.

Silva Jr. AI, Pereira HMG, A. Casilli A, Conceicão FC, Neto FRA (2009) Journal of Chromatography A 1216: 2913-2922.

Simmons MC, Snyder LR (1958) Analytical Chemistry 30: 32 .

Tranchida PQ, Dugo P, Dugo G, Mondello L (2004) Journal Chromatography A 1054: 3.

Tranchida PQ, Purcaro G, Conte L, Dugo P, Dugo G, Mondello L (2009) Journal of Chromatography A 1216: 7301-7306.

Tranchida PQ, Sciarrone D, Dugo P, Mondello L (2012) Analytica Chimica Acta 716: 66-75.

Van Stee LLP, Beens J, Vreuls RJJ, Brinkman UAT (2003) Journal of Chromatography A 1019: 89-99.

Weldegergis BT, Crouch AM, Górecki T, Villiers A (2011) Analytica Chimica Acta 701: 98-111.

Williams A, Ryan D, Guasca AO, Marriott P, Pang E (2004) Journal of Chromatography B 817: 97-107.

Wilson, Wilson's (2009) Comprehensive analytical chemistry, Elsevier, Amsterdam, The Netherlands.

Yang W, Zhi-Qiang Y, Xiang-Fan L, Jia-Liang F, Dong-Ping Z, Guo-Fa R, Guo-Ying S, Jia-Mo F (2012) Chinese Journal of Analytical Chemistry 40: 1187-1193.

Zapadlo M, Krupčík J, Kovalczuk T, Májek P, Špánik I, Armstrong DW, Sandra P (2010) Journal of Chromatography A 1217: 5859-5867. 